\title{
Van der Woude syndrome
}

INSERM

\section{Source}

INSERM. (1999). Orphanet: an online rare disease and orphan drug data base. Van der Woude syndrome. ORPHA:888

Van der Woude syndrome (VWS) is a rare congenital genetic dysmorphic syndrome characterized by paramedian lower-lip fistulae, cleft lip with or without cleft palate, or isolated cleft palate. 